\title{
Erratum to: Reactive trajectories and the transition path process
}

\author{
Jianfeng Lu • James Nolen
}

Published online: 9 April 2014

(C) Springer-Verlag Berlin Heidelberg 2014

\section{Erratum to: Probab. Theory Relat. Fields DOI 10.1007/s00440-014-0547-y}

The author would like to correct the errors in the publication of the original article. The corrected details are given below for your reading.

In the last line of the abstract, "Weinan and Vanden-Eijnden" should be "E and VandenEijnden".

In the third line of the last paragraph of the second page of the paper, "Weinan and Vanden-Eijnden" should be "E and Vanden-Eijnden".

In the first sentence of Section 1.6, "Weinan and Vanden-Eijnden" should be "E and Vanden-Eijnden".

In both references 15 and 16 of the paper, "Weinan, E." should be changed to "E, W.".

The online version of the original article can be found under doi:10.1007/s00440-014-0547-y.

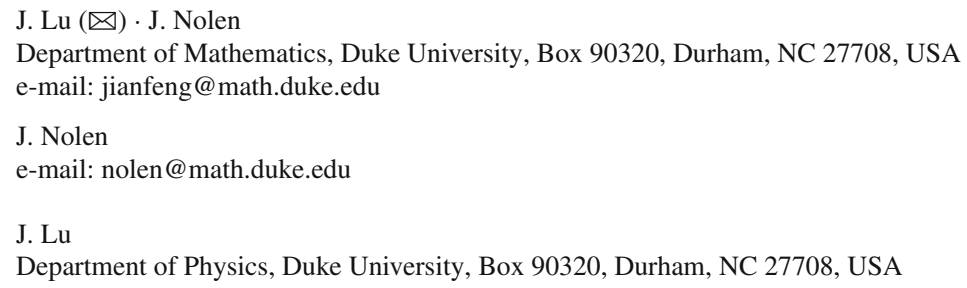

